\title{
ON THE GRAPH OF MODULES OVER COMMUTATIVE RINGS
}

\author{
H. ANSARI-TOROGHY AND SH. HABIBI
}

\begin{abstract}
Let $M$ be a module over a commutative ring and let $\operatorname{Spec}(M)$ be the collection of all prime submodules of $M$. We topologize $\operatorname{Spec}(M)$ with quasi-Zariski topology and, for a subset $T$ of $\operatorname{Spec}(M)$, we introduce a new graph $G\left(\tau_{T}^{*}\right)$, called the quasi-Zariski topology-graph. It helps us to study algebraic (respectively, topological) properties of $M$ (respectively, $\operatorname{Spec}(M)$ ) by using graph theoretical tools. Also, we study the annihilating-submodule graph and investigate the relation between these two graphs.
\end{abstract}

1. Introduction. Throughout this paper, $R$ is a commutative ring with a non-zero identity and $M$ is a unital $R$-module. By $N \leq M$ (respectively $N<M$ ) we mean that $N$ is a submodule (respectively proper submodule) of $M$ and $\Lambda(M)$ is the set of all non-zero submodules of $M$. For any pair of submodules $N \subseteq L$ of $M$ and any element $m$ of $M$, we denote $L / N$ and the residue class of $m$ modulo $N$ in $M / N$ by $\bar{L}$ and $\bar{m}$, respectively.

For a submodule $N$ of $M$, the colon ideal of $M$ into $N$ is defined by $(N: M)=\{r \in R \mid r M \subseteq N\}=\operatorname{Ann}(M / N)$. Further if $I$ is an ideal of $R$, the submodule $\left(N:_{M} I\right)$ is defined by $\{m \in M: \Im \subseteq N\}$. Moreover, $\mathbb{N}, \mathbb{Z}$ and $\mathbb{Q}$ denote the set of positive integers, the ring of integers, and the field of rational numbers, respectively.

For a subset $T$ of $\operatorname{Spec}(M), \Im(T)$ is the intersection of all members of $T$.

A prime submodule of $M$ is a submodule $P \neq M$ such that, whenever $r e \in P$ for some $r \in R$ and $e \in M$, we have $r \in(P: M)$ or $e \in P[\mathbf{1 3}]$.

2010 AMS Mathematics subject classification. Primary 13C13, 13 C99.

Keywords and phrases. Prime submodule, top module, quasi-Zariski topology, graph, vertices, annihilating-submodule.

Received by the editors on August 4, 2013, and in revised form on July 11, 2014. DOI: $10.1216 /$ RMJ-2016-46-3-729 
The prime spectrum (or simply, the spectrum) of $M$ is the set of all prime submodules of $M$ and denoted by $\operatorname{Spec}(M)$. Also, the set of all maximal submodules of $M$ is denoted by $\operatorname{Max}(M)$.

The prime radical $\sqrt{N}$ is defined to be the intersection of all prime submodules of $M$ containing $N$, and in the case of $N$ is not contained in any prime submodule, $\sqrt{N}$ is defined to be $M$. Note that the intersection of all prime submodule $M$ is denoted by $\operatorname{rad}(M)$.

The quasi-Zariski topology on $X:=\operatorname{Spec}(M)$ is described as follows: put $V^{*}(N)=\{P \in X: P \supseteq N\}$ and $\xi^{*}(M)=\left\{V^{*}(N): N\right.$ is a submodule of $M\}$. Then there exists a topology $\tau^{*}$ on $X$ having $\xi^{*}$ as the set of closed subsets of $\operatorname{Spec}(M)$ if and only if $\xi^{*}$ is closed under the finite union. When this is the case, $\tau_{M}^{*}$ is called the quasi-Zariski topology on $\operatorname{Spec}(M)$ and $M$ is called a top module [14].

If $\operatorname{Spec}(M) \neq \emptyset$, the mapping $\psi: \operatorname{Spec}(M) \rightarrow \operatorname{Spec}(R / \operatorname{Ann}(M))$ such that $\psi(P)=(P: M) / \operatorname{Ann}(M)=\overline{(P: M)}$ for every $P \in$ $\operatorname{Spec}(M)$, is called the natural map of $\operatorname{Spec}(M)[6]$.

A topological space $X$ is said to be connected if there does not exist a pair $U, V$ of disjoint non-empty open sets of $X$ whose union is $X$. A topological space $X$ is irreducible if, for any decomposition $X=X_{1} \cup X_{2}$ with closed subsets $X_{i}$ of $X$ with $i=1$, 2, we have $X=X_{1}$ or $X=X_{2}$. A subset $X^{\prime}$ of $X$ is connected (respectively irreducible) if it is connected (respectively irreducible) as a subspace of $X$.

The zero-divisor graph of $R, \Gamma(R)$, is a graph with the vertex set $Z(R) \backslash\{0\}$, the set of nonzero zero-divisors of $R$, and two distinct vertices $x$ and $y$ are adjacent if and only if $x y=0$. The concept of the zero-divisor graph was first introduced by Beck (see [7]). Since many properties of a ring are closely tied to the behavior of its ideals, it is valuable to replace the vertices of the zero-divisor graph by the non-zero annihilator ideals. The idea of a graph, whose vertices are a subset of ideals of a ring, was introduced recently in [10]. They defined $A G(R)$, the annihilating-ideal graph of $R$, to be a graph whose vertices are ideals of $R$ with non-zero annihilators and in which two vertices $I$ and $J$ are adjacent if and only if $I J=0$. 
Let $N$ and $L$ be submodules of $M$. Then the product of $N$ and $L$ is defined by $(N: M)(L: M) M$ and denoted by $N L$, and clearly $N^{k}=(N: M)^{k} M($ see $[3])$.

In [4], the present authors generalized the above idea, introduced the annihilating-submodule graph $A G(M)$ and investigated some of its related properties. The (undirected) graph $A G(M)$ is a graph with vertices $V(A G(M))=\{N \leq M$ : there exists a non-zero proper submodule $L$ of $M$ with $N L=0\}$, where distinct vertices $N, L$ are adjacent if and only if $N L=0$.

As we know, the closed subset $V^{*}(N)$, where $N$ is a submodule of $M$, plays an important role in the quasi-Zariski topology on $\operatorname{Spec}(M)$. Our main purpose in this article is to employ these sets and define a new graph $G\left(\tau_{T}^{*}\right)$, called the quasi-Zariski topology-graph. By using this graph, we study algebraic (respectively, topological) properties of $M$ (respectively, $\operatorname{Spec}(M))$. Further, we investigate the relationship between $G\left(\tau_{T}^{*}\right)$ and $A G(M / \Im(T))$, where $T$ denotes a non-empty subset of $\operatorname{Spec}(M)$ and $\Im(T)$ is the intersection of all members of $T$.

$G\left(\tau_{T}^{*}\right)$ is an undirected graph with vertices $V\left(G\left(\tau_{T}^{*}\right)\right)=\{N<$ $M$ : there exists $K<M$ such that $V^{*}(N) \cup V^{*}(K)=T$ and $V^{*}(N), V^{*}(K) \neq T$ \}, where $T$ is a non-empty subset of $\operatorname{Spec}(M)$ and distinct vertices $N$ and $L$ are adjacent if and only if $V^{*}(N) \cup V^{*}(L)=T$ (see Definition 2.1).

Let $M$ be a top module. In Section 2 of this article, among other results, it is shown that the quasi-Zariski topology-graph $G\left(\tau_{T}^{*}\right)$ is connected and $\operatorname{diam}\left(G\left(\tau_{T}^{*}\right)\right) \leq 3$. Further if $G\left(\tau_{T}^{*}\right)$ contains a cycle, then $\operatorname{gr}\left(G\left(\tau_{T}^{*}\right)\right) \leq 4$ (see Theorem 2.6). Also, it is shown that $G\left(\tau_{T}^{*}\right)$ has a bipartite subgraph (see Theorem 2.14).

In Section 3, we explore more properties of $A G(M)$. In Proposition 3.4 , we show that if $M$ is a non-simple semisimple $R$-module, then every non-zero proper submodule of $M$ is a vertex. In Theorem 3.7, we provide some useful characterizations for those modules $M$ for which $A G(M)=K_{\alpha}$, where $|\Lambda(M)|=\alpha$.

In Section 4, the relationship between $G\left(\tau_{T}^{*}\right)$ and $A G(M / \Im(T))$ is investigated. We show that, if $N$ and $L$ are non-zero proper submodules of $M$ which are adjacent in $G\left(\tau_{T}^{*}\right)$, then $\sqrt{N} / \Im(T)$ and $\sqrt{L} / \Im(T)$ are adjacent in $A G(M / \Im(T)$ ) (see Proposition 4.5). Also we show that, if $M$ is a finitely generated module and $G\left(\tau_{T}^{*}\right) \neq \emptyset$, then $A G(M / \Im(T))$ is 
isomorphic with a subgraph of $G\left(\tau_{T}^{*}\right)$. Further, we prove that, if $M$ is a fully semiprime module, then $G\left(\tau_{T}^{*}\right)$ is isomorphic with a subgraph of $A G(M / \Im(T))$ (see Theorem 4.6).

Let us introduce some graphical notation that is used in what follows. A graph $G$ is an ordered triple $\left(V(G), E(G), \psi_{G}\right)$ consisting of a nonempty set of vertices, $V(G)$, a set $E(G)$ of edges, and an incident function $\psi_{G}$ that associates an unordered pair of distinct vertices with each edge. The edge $e$ joins $x$ and $y$ if $\psi_{G}(e)=\{x, y\}$, and we say $x$ and $y$ are adjacent. The degree $d_{G}(x)$ of a vertex $x$ is the number of edges incident with $x$. A path in graph $G$ is a finite sequence of vertices $\left\{x_{0}, x_{1}, \ldots, x_{n}\right\}$, where $x_{i-1}$ and $x_{i}$ are adjacent for each $1 \leq i \leq n$ and we denote $x_{i-1}-x_{i}$ for an existing edge between $x_{i-1}$ and $x_{i}$. The number of edges crossed to get from $x$ to $y$ in a path is called the length of the path. A graph $G$ is connected if a path exists between any two distinct vertices. For distinct vertices $x$ and $y$ of $G$, let $d(x, y)$ be the length of the shortest path from $x$ to $y$ and, if there is no such path, then $d(x, y)=\infty$. The diameter of $G$ is $\operatorname{diam}(G)=\sup \{d(x, y): x, y \in V(G)\}$. The girth of $G$, denoted by $\operatorname{gr}(G)$, is the length of the shortest cycle in $G$ and, if $G$ contains no cycles, then $\operatorname{gr}(G)=\infty$ (see [1]).

A graph $H$ is a subgraph of $G$ if $V(H) \subseteq V(G), E(H) \subseteq E(G)$ and $\psi_{H}$ is the restriction of $\psi_{G}$ to $E(H)$. We denote the complete graph on $n$ vertices by $K_{n}$. A bipartite graph is a graph whose vertices can be divided into two disjoint sets $U$ and $V$ such that every edge connects a vertex in $U$ to one in $V$; that is, $U$ and $V$ are each independent sets and complete bipartite graphs on $n$ and $m$ vertices, denoted by $K_{n, m}$, where $V$ and $U$ are of size $n$ and $m$, respectively, and $E(G)$ connects every vertex in $V$ with all vertices in $U$ (see [16]).

In the rest of this article, $M$ denotes a top module, $T$ a non-empty subset of $\operatorname{Spec}(M), \Im(T)$ is the intersection of all members of $T, \widehat{M}$ represents the $R$-module $M / \Im(T)$, and for a submodule $N$ of $M$, $\widehat{N}=N / \Im(T)$, where $\Im(T) \subseteq N$, is a submodule of $\widehat{M}$.

\section{The qausi-Zariski topology-graph.}

Definition 2.1. We define a quasi-Zariski topology-graph $G\left(\tau_{T}^{*}\right)$ with vertices $V\left(G\left(\tau_{T}^{*}\right)\right)=\left\{N<M\right.$ : there exists $K<M$ such that $V^{*}(N) \cup$ 
$V^{*}(K)=T$ and $\left.V^{*}(N), V^{*}(K) \neq T\right\}$, where distinct vertices $N$ and $L$ are adjacent if and only if $V^{*}(N) \cup V^{*}(L)=T$.

Notation 2.2. By [14, Lemma 2.1], if $M$ is a top module, then for every pair of submodules $N$ and $L$ of $M$, we have $V^{*}(N) \cup V^{*}(L)=$ $V^{*}(\sqrt{N}) \cup V^{*}(\sqrt{L})=V^{*}(\sqrt{N} \cap \sqrt{L})$.

Proposition 2.3. The following statements hold.

(i) $G\left(\tau_{T}^{*}\right) \neq \emptyset$ if and only if $T$ is closed and is not an irreducible subset of $\operatorname{Spec}(M)$.

(ii) $G\left(\tau_{T}^{*}\right) \neq \emptyset$ if and only if $T=V^{*}(\Im(T))$ and $T$ is not an irreducible subset of $\operatorname{Spec}(M)$.

(iii) $G\left(\tau_{T}^{*}\right) \neq \emptyset$ if and only if $T=V^{*}(\Im(T))$ and $\Im(T)$ is not a prime submodule of $M$.

\section{Proof.}

(i) Straightforward.

(ii) Suppose that $G\left(\tau_{T}^{*}\right) \neq \emptyset$. By part (i), it is enough to show that $T=V^{*}(\Im(T))$ which is a closed set. Clearly, $T \subseteq V^{*}(\Im(T))$. Next, let $V^{*}(N)$ be any closed subset of $\operatorname{Spec}(M)$ containing $T$. Then $P \supseteq N$ for every $P \in T$ so that $\Im(T) \supseteq N$. Hence, for every $Q \in V^{*}(\Im(T))$ and $Q \supseteq \Im(T) \supseteq N$, namely, $V^{*}(\Im(T)) \subseteq V^{*}(N)$, it follows that $V^{*}(\Im(T))$ is the smallest closed subset of $\operatorname{Spec}(M)$ containing $T$. Hence, $V^{*}(\Im(T))=T$.

(iii) It follows from part (ii) and [8, Theorem 3.4].

Example 2.4. Set $R:=\mathbb{Z}$ and $M:=\mathbb{Z} \oplus \mathbb{Z}\left(p^{\infty}\right)$, where $p$ is a prime integer of $\mathbb{Z}$. Then, by [6, Examples 3.1], $\operatorname{Max}(M)=\left\{p_{i} \mathbb{Z} \oplus \mathbb{Z}\left(p^{\infty}\right): i \in\right.$ $\mathbb{N}\}, \operatorname{Spec}(M)=\operatorname{Max}(M) \cup\left\{(\mathbf{0}) \oplus \mathbb{Z}\left(p^{\infty}\right)\right\}$, where $p_{i}$ is a prime number for every $i \in \mathbb{N}$, and $M$ is a top module. We have $V^{*}\left((\mathbf{0}) \oplus \mathbb{Z}\left(p^{\infty}\right)\right)=$ $\operatorname{Spec}(M)$. Hence $\operatorname{Spec}(M)$ is irreducible and $G\left(\tau_{\operatorname{Spec}(M)}^{*}\right)=\emptyset$.

Example 2.5. Set $R:=\mathbb{Z}$ and $M:=\mathbb{Q} \oplus\left(\oplus_{i \in \mathbb{N}} \mathbb{Z} / p_{i} \mathbb{Z}\right)$. Then by [6, Examples 3.1],

$$
\begin{aligned}
\operatorname{Max}(M) & =\left\{\mathbb{Q} \oplus\left(\oplus_{i \in \mathbb{N}, i \neq j} \mathbb{Z} / p_{i} \mathbb{Z}\right)\right\}, \\
\operatorname{Spec}(M) & =\operatorname{Max}(M) \cup\left\{(\mathbf{0}) \oplus\left(\oplus_{i \in \mathbb{N}} \mathbb{Z} / p_{i} \mathbb{Z}\right)\right\},
\end{aligned}
$$


and $M$ is a top module. Now, $\mathbb{Q} \oplus(\mathbf{0})$ and $\left\{(\mathbf{0}) \oplus\left(\oplus_{i \in \mathbb{N}} \mathbb{Z} / p_{i} \mathbb{Z}\right)\right\}$ are adjacent in $G\left(\tau_{\operatorname{Spec}(M)}^{*}\right)$ so that $G\left(\tau_{\operatorname{Spec}(M)}^{*}\right) \neq \emptyset$.

The following theorem illustrates some graphical parameters.

Theorem 2.6. The quasi-Zariski topology-graph $G\left(\tau_{T}^{*}\right)$ is connected and $\operatorname{diam}\left(G\left(\tau_{T}^{*}\right)\right) \leq 3$. Moreover, if $G\left(\tau_{T}^{*}\right)$ contains a cycle, then $\operatorname{gr}\left(G\left(\tau_{T}^{*}\right)\right) \leq 4$.

Proof. Suppose $N, K \in V\left(G\left(\tau_{T}^{*}\right)\right)$ and they are not adjacent. Then $V^{*}(N) \cup V^{*}(K) \neq T$, so there exist $L, V \in V\left(G\left(\tau_{T}^{*}\right)\right)$ with $V^{*}(\sqrt{N} \cap$ $\sqrt{L})=V^{*}(\sqrt{K} \cap \sqrt{V})=T$. If $L=V$, then $N-L-K$ is a path of length 2. Thus, we assume that $L \neq V$. If $V^{*}(\sqrt{L} \cap \sqrt{V})=T$, then $N-L-V-K$ is a path of length 3. If $V^{*}(\sqrt{L} \cap \sqrt{V}) \neq T$, then $N-\sqrt{L} \cap \sqrt{V}-K$ is a path of length 2 (if $N=\sqrt{L} \cap \sqrt{V}$, then $V^{*}(N) \cup V^{*}(K)=V^{*}(L) \cup V^{*}(V) \cup V^{*}(K)$ so that $T=V^{*}(\sqrt{V} \cap$ $\sqrt{K})=V^{*}(\sqrt{L} \cap \sqrt{V} \cap \sqrt{K})$. Thus, $V^{*}(\sqrt{N}) \cap V^{*}(\sqrt{K})=T$, a contradiction. Similarly, we have $K \neq \sqrt{L} \cap \sqrt{V})$. Now suppose that $\operatorname{gr}\left(G\left(\tau_{T}^{*}\right)\right)>4$. We can assume that $\operatorname{gr}\left(G\left(\tau_{T}^{*}\right)\right)=k$, where $k>4$. Then $N_{1}-N_{2}-N_{3}-N_{4}-N_{5}-\cdots-N_{k-1}-N_{k}-N_{1}$ is a cycle of length $k$. Clearly, $V^{*}\left(N_{2}\right) \cup V^{*}\left(N_{k-1}\right) \neq T$. Now one can see that $N_{1}-\sqrt{N_{2}} \cap \sqrt{N_{k-1}}-N_{k}-N_{1}$ is a 3-cycle, a contradiction. So we have $\operatorname{gr}\left(G\left(\tau_{T}^{*}\right)\right) \leq 4$. Hence, the proof is complete.

Proposition 2.7. Let $M$ be an $R$-module, and let $\psi: \operatorname{Spec}(M) \rightarrow$ $\operatorname{Spec}(R / \operatorname{Ann}(M))$ be the natural map. Suppose $\operatorname{Spec}(M)$ is homeomorphic to $\operatorname{Spec}(R / \operatorname{Ann}(M))$ under $\psi$. Let $(N: M) M$ and $(L: M) M$ be adjacent in $G\left(\tau_{T}^{*}\right)$, and let $T^{\prime}=\{\overline{(P: M)}: P \in T\}$. Then $\overline{(N: M)}$ and $\overline{(L: M)}$ are adjacent in $G\left(\tau_{T^{\prime}}^{*}\right)$. Conversely, if $\bar{I}$ and $\bar{J}$ are adjacent in $G\left(\tau_{T^{\prime}}^{*}\right)$, then $I M$ and $J M$ are adjacent in $G\left(\tau_{T}^{*}\right)$.

Proof. Since $\psi$ is injective, $\psi^{-1}\left(T^{\prime}\right)=T$. Also we have $V^{*}((N$ : $M) M) \cup V^{*}((L: M) M)=T$. Hence,

$$
\psi\left(V^{*}((N: M) M)\right) \cup \psi\left(V^{*}((L: M) M)\right)=T^{\prime} .
$$

This implies that $V(\overline{N: M}) \cup V(\overline{L: M})=T^{\prime}$ (note that $V^{*}((N$ : $\left.M) M)=T \Leftrightarrow V(\overline{N: M})=T^{\prime}\right)$. Conversely, suppose $V(\bar{I}) \cup V(\bar{J})=$ 
$T^{\prime}$. Then $\psi^{-1}(V(\bar{I})) \cup \psi^{-1}(V(\bar{J}))=T$ so that $V^{*}(I M) \cup V^{*}(J M)=T$ (note that $V^{*}(\bar{I})=T^{\prime} \Leftrightarrow V^{*}(I M)=T$ ).

Lemma 2.8. Let $G\left(\tau_{T}^{*}\right) \neq \emptyset$ and let $P \in T$. Then $P$ is a vertex if either of the following statements holds.

(i) There exists a subset $T^{\prime}$ of $T$ such that $P \in T^{\prime}, V^{*}\left(\cap_{Q \in T^{\prime}} Q\right)=T$, and $V^{*}\left(\cap_{Q \in T^{\prime}, Q \neq P} Q\right) \neq T$.

(ii) For a submodule $N$ of $M, N \in V\left(G\left(\tau_{T}^{*}\right)\right)$ and $\sqrt{N} \cap P \notin$ $V\left(G\left(\tau_{T}^{*}\right)\right)$.

Proof. Straightforward.

The following theorem shows the situations in which $T$ contains some vertices.

Theorem 2.9. Suppose $T$ is a finite set and $G\left(\tau_{T}^{*}\right) \neq \emptyset$. Then

(i) $T \cap V\left(G\left(\tau_{T}^{*}\right)\right) \neq \emptyset$.

(ii) If $T \subseteq \operatorname{Max}(M)$, then every $P \in T$ is a vertex.

(iii) If $P \in T \cap \operatorname{Min}(M)$, then $P$ is a vertex.

Proof.

(i) Let $P \in T$. Then we have $V^{*}(P) \cup V^{*}\left(\cap_{Q \in T, Q \neq P} Q\right)=T$. If $V^{*}\left(\cap_{Q \in T, Q \neq P} Q\right) \neq T$, then $P$ is a vertex. Otherwise, we have $V^{*}\left(\cap_{Q \in T, Q \neq P} Q\right)=T$. Since $T$ is not irreducible, there exists a non-empty subset $T^{\prime}$ of $T$ and $P^{\prime} \in T^{\prime}$ such that

$$
V^{*}\left(\cap_{P \in T \backslash T^{\prime}} P\right) \neq T \quad \text { and } \quad V^{*}\left(\cap_{P \in\left(T \backslash T^{\prime}\right) \cup\left\{P^{\prime}\right\}} P\right)=T .
$$

Thus, $P^{\prime} \in T \cap V\left(G\left(\tau_{T}^{*}\right)\right)$.

(ii) Clearly, $V^{*}(P) \cup V^{*}\left(\cap_{Q \in T, Q \neq P} Q\right)=T$ and $V^{*}\left(\cap_{Q \in T, Q \neq P} Q\right) \neq T$.

(iii) Clearly, $V^{*}(P) \cup V^{*}\left(\cap_{Q \in T, Q \neq P} Q\right)=T$ and $V^{*}\left(\cap_{Q \in T, Q \neq P} Q\right) \neq T$.

Example 2.10. Consider Example 2.4. If $|T| \geq 2$ and $T \subseteq\left\{p_{1} \mathbb{Z} \oplus\right.$ $\left.\mathbb{Z}\left(p^{\infty}\right), \ldots, p_{n} \mathbb{Z} \oplus \mathbb{Z}\left(p^{\infty}\right)\right\}$, then every element of $T$ is a vertex. Moreover, in Example 2.5, if $|T| \geq 2$ and 


$$
T \subseteq\left\{\mathbb{Q} \oplus\left(\oplus_{i \in \mathbb{N}, i \neq 1} \mathbb{Z} / p_{i} \mathbb{Z}\right), \ldots, \mathbb{Q} \oplus\left(\oplus_{i \in \mathbb{N}, i \neq n} \mathbb{Z} / p_{i} \mathbb{Z}\right)\right\},
$$

then every element of $T$ is a vertex.

Definition 2.11. We define a subgraph $G_{d}\left(\tau_{T}^{*}\right)$ of $G\left(\tau_{T}^{*}\right)$ with vertices $V\left(\left(G_{d}\left(\tau_{T}^{*}\right)\right)\right)=\left\{N<M\right.$ : there exists $L<M$ such that $V^{*}(N) \cup$ $V^{*}(L)=T, V^{*}(N), V^{*}(L) \neq T$ and $\left.V^{*}(N) \cap V^{*}(L)=\emptyset\right\}$, where distinct vertices $N$ and $L$ are adjacent if and only if $V^{*}(N) \cup V^{*}(L)=$ $T$ and $V^{*}(N) \cap V^{*}(L)=\emptyset$. It is clear that the degree of every $N \in V\left(\left(G_{d}\left(\tau_{T}^{*}\right)\right)\right)$ is the number of submodules $K$ of $M$ such that $V^{*}(L)=V^{*}(K)$, where $L$ is adjacent to $N$.

We need the following remark.

Remark 2.12. We recall that the Zariski topology on $\operatorname{Spec}(M)$ is the topology $\tau_{M}$ described by taking the set $Z(M)=\{V(N): N \leq M\}$ as the set of closed sets of $\operatorname{Spec}(M)$, where $V(N)=\{P \in \operatorname{Spec}(M):(P$ : $M) \supseteq(N: M)\}$ [12]. If $M$ is a multiplication module, then $\tau_{M}=\tau_{M}^{*}$ by $[\mathbf{1 4}$, Theorem 3.5].

Proposition 2.13. The following statements hold.

(i) $G_{d}\left(\tau_{T}^{*}\right) \neq \emptyset$ if and only if $T=V^{*}(\Im(T))$ and $T$ is disconnected.

(ii) Suppose $\widehat{M}$ is an Artinian module and $T$ is closed. Then $G_{d}\left(\tau_{T}^{*}\right)=$ $\emptyset$ if and only if $R / \operatorname{Ann}(\widehat{M})$ contains no idempotent other than $\overline{0}$ and $\overline{1}$.

Proof.

(i) Straightforward.

(ii) Since $\widehat{M}$ is an Artinian module, then $\widehat{M} / \operatorname{rad}(\widehat{M})$ is a Noetherian module by [8, Corollary 2.30]. As $\widehat{M} / \operatorname{rad}(\widehat{M})$ is a finitely generated top module, it is a multiplication module by [14, Theorem 3.5]. It follows that $\tau_{\hat{M} / \operatorname{rad}(\hat{M})}=\tau_{\hat{M} / \operatorname{rad}(\hat{M})}^{*}$ by Remark 2.12. So $\tau_{\hat{M}}=\tau_{\hat{M}}^{*}$ because $\widehat{M}$ and $\widehat{M} / \operatorname{rad}(\widehat{M})$ are homeomorphic by Lemma 4.1. Also, the natural map of $\widehat{M} / \operatorname{rad}(\widehat{M})$ is surjective (for, $\widehat{M} / \operatorname{rad}(\widehat{M})$ is finitely 
generated). Hence, the natural map of $\widehat{M}$ is surjective by the above arguments. Now the result follows from [12, Corollary 3.8].

Theorem 2.14. $G_{d}\left(\tau_{T}^{*}\right)$ is a bipartite graph.

Proof. At first we assume that $G_{d}\left(\tau_{T}^{*}\right)$ contains a cycle. We show that $\operatorname{gr}\left(G_{d}\left(\tau_{T}^{*}\right)\right) \leq 4$. Now suppose that $\operatorname{gr}\left(G_{d}\left(\tau_{T}^{*}\right)\right)>4$. We can assume that $\operatorname{gr}\left(G_{d}\left(\tau_{T}^{*}\right)\right)=k$, where $k>4$. Then $N_{1}-N_{2}-N_{3}-N_{4}-$ $N_{5}-\cdots-N_{k-1}-N_{k}-N_{1}$ is a cycle of length $k$. Clearly, $V^{*}\left(N_{k-1}\right)=$ $V^{*}\left(N_{1}\right)$. Hence, one can see that $N_{1}-N_{2}-N_{3}-\cdots-N_{k-2}-N_{1}$ is a cycle, a contradiction. So we have $\operatorname{gr}\left(G_{d}\left(\tau_{T}^{*}\right)\right) \leq 4$. Now, by $[\mathbf{1 6}$, Proposition 1.6.1], $G$ is a bipartite graph if and only if it does not contain an odd cycle. Hence, by Theorem 2.6, it is enough to show that $\operatorname{gr}\left(G_{d}\left(\tau_{T}^{*}\right)\right) \neq 3$. Suppose $N-L-K-N$ is a 3 -cycle. Then

$$
\begin{aligned}
\emptyset & =\left(V^{*}(N) \cap V^{*}(L)\right) \cup\left(V^{*}(N) \cap V^{*}(K)\right) \\
& =V^{*}(N) \cap\left(V^{*}(L) \cup V^{*}(K)\right)=V^{*}(N) \cap T=V^{*}(N) .
\end{aligned}
$$

Hence, $V(N)=\emptyset$, a contradiction.

Corollary 2.15. By Theorem 2.14, if $G_{d}\left(\tau_{T}^{*}\right)$ contains a cycle, then $\operatorname{gr}\left(G_{d}\left(\tau_{T}^{*}\right)\right)=4$.

Example 2.16. Set $R:=\mathbb{Z}$ and $M:=\mathbb{Z} / 12 \mathbb{Z}$. So $\operatorname{Spec}(M)=$ $\operatorname{Max}(M)=\{2 \mathbb{Z} / 12 \mathbb{Z}, 3 \mathbb{Z} / 12 \mathbb{Z}\}$. Set $T:=\operatorname{Spec}(M)$. Clearly, $G\left(\tau_{T}^{*}\right)=$ $G_{d}\left(\tau_{T}^{*}\right)$ is a bipartite graph and $\mathbb{Z} /\left(\cap_{P \in T} P: M\right) \cong \mathbb{Z} / 6 \mathbb{Z}$ contains idempotents other than $\overline{0}$ and $\overline{1}$.

Example 2.17. Set $R:=\mathbb{Z}$ and $M:=\mathbb{Z} / 30 \mathbb{Z}$. So $\operatorname{Spec}(M)=$ $\operatorname{Max}(M)=\{2 \mathbb{Z} / 30 \mathbb{Z}, 3 \mathbb{Z} / 30 \mathbb{Z}, 5 \mathbb{Z} / 30 \mathbb{Z}\}$. Set $T:=\operatorname{Spec}(M)$. Clearly, $G_{d}\left(\tau_{T}^{*}\right)$ is a bipartite graph and $\mathbb{Z} /\left(\cap_{P \in T} P: M\right) \cong \mathbb{Z} / 30 \mathbb{Z}$ contains idempotents other than $\overline{0}$ and $\overline{1}$.

The above example shows that $G_{d}\left(\tau_{T}^{*}\right)$ is not always connected.

Proposition 2.18. The following statements hold. 
(i) $G_{d}\left(\tau_{T}^{*}\right)$ with two parts $U$ and $V$ is a complete bipartite graph if and only if for every $N, L \in U$ (respectively in $V), V^{*}(N)=V^{*}(L)$.

(ii) $G_{d}\left(\tau_{T}^{*}\right)$ is connected if and only if it is a complete bipartite graph.

Proof. Use the fact that if $N$ and $L$ are two vertices, then $d(N, L)=$ 2 if and only if $V^{*}(N)=V^{*}(L)$.

We end this section with the following question.

Question 2.19. Let $G\left(\tau_{T}^{*}\right) \neq \emptyset$, where $T$ is an infinite subset of $\operatorname{Spec}(M)$. Is $T \cap V\left(G\left(\tau_{T}^{*}\right)\right) \neq \emptyset$ ?

3. The annihilating-submodule graph. As we mentioned before, $A G(M)$ is a graph with vertices $V(A G(M))=\{N \leq M: N L=0$ for some $0 \neq L<M\}$, where distinct vertices $N$ and $L$ are adjacent if and only if $N L=0$ (here we recall that the product of $N$ and $L$ is defined by $(N: M)(L: M) M)$.

The following results reflect some basic properties of the annihilatingsubmodule graph of a module.

Proposition A ([4, Proposition 3.2]). Let $N$ be a non-zero proper submodule of $M$.

(i) $N$ is a vertex in $A G(M)$ if $\operatorname{Ann}(N) \neq \operatorname{Ann}(M)$ or $\left(0:_{M}(N\right.$ : $M)) \neq 0$.

(ii) $N$ is a vertex in $A G(M)$, where $M$ is a multiplication module, if and only if $\left(0:_{M}(N: M)\right) \neq 0$.

Remark 3.1. In the annihilating-submodule graph $A G(M), M$ itself can be a vertex. In fact $M$ is a vertex if and only if every non-zero submodule is a vertex if and only if there exists a non-zero proper submodule $N$ of $M$ such that $(N: M)=\operatorname{Ann}(M)$. For example, for every submodule $N$ of $\mathbb{Q}$ (as a $\mathbb{Z}$-module), $(N: \mathbb{Q})=0$. Hence, $\mathbb{Q}$ is a vertex in $A G(\mathbb{Q})$.

Theorem B ([4, Theorem 3.3]). Assume that $M$ is not a vertex. Then the following hold.

(i) $A G(M)$ is empty if and only if $M$ is a prime module. 
(ii) A non-zero submodule $N$ of $M$ is a vertex if and only if $(0: M$ $(N: M)) \neq 0$.

Theorem $\mathbf{C}$ ([4, Theorem 3.4]). The annihilating-submodule graph $A G(M)$ is connected and $\operatorname{diam}(A G(M)) \leq 3$. Moreover, if $A G(M)$ contains a cycle, then $\operatorname{gr}(A G(M)) \leq 4$.

Lemma 3.2. Let $M$ be an $R$-module and $\operatorname{Ann}(M)$ a prime ideal. Then $\operatorname{diam}(A G(M)) \leq 2$.

Proof. Suppose $N$ and $L$ are adjacent in $A G(M)$. Then $(N: M)=$ $\operatorname{Ann}(M)$ or $(L: M)=\operatorname{Ann}(M)$. Assume that $(N: M)=\operatorname{Ann}(M)$. So every non-zero submodule $M$ is a vertex and adjacent to $N$. Hence, $\operatorname{diam}(A G(M)) \leq 2$.

Proposition 3.3. The following statements hold.

(i) Let $M=R m$ be a cyclic $R$-module. Then $M$ is not a vertex.

(ii) Let $M=M_{1} \oplus M_{2}$, where $M_{1}, M_{2}$ are non-zero $R$-submodules of $M$. Then every non-zero submodule of $M_{1}$ is adjacent to every non-zero submodule of $M_{2}$.

(iii) Assume that $A G(M)=\emptyset$. Then module $M$ is an indecomposable module.

Proof.

(i) This follows from Remark 3.1 and the fact that every cyclic $R$ module is multiplication.

(ii) Let $0 \neq N \leq M_{1}$ and $0 \neq K \leq M_{2}$. Clearly, $(N \oplus(\mathbf{0}): M)=$ $\left(N: M_{1}\right) \cap\left(0: M_{2}\right)$. Hence, $(N \oplus(\mathbf{0}): M) \subseteq\left(0: M_{2}\right)$. Similarly, $((\mathbf{0}) \oplus K: M) \subseteq\left(0: M_{1}\right)$. Therefore, $(N \oplus(\mathbf{0}))((\mathbf{0}) \oplus K)=0$. This in turn implies that $N$ and $K$ are adjacent in $A G(M)$.

(iii) The proof follows from part (ii).

We allow $\alpha$ to be infinite cardinal, where $\alpha=|\Lambda(M)|$. (We recall that $\Lambda(M)$ is the set of all non-zero submodules of $M$.)

Proposition 3.4. The following statements hold. 
(i) Let $M$ be a non-simple semisimple $R$-module. Then every nonzero proper submodule of $M$ is a vertex.

(ii) Let $M$ be a non-simple homogeneous semisimple $R$-module. Then $A G(M)=K_{\alpha}$.

(iii) Let $M$ be a prime module with a non-zero socle. Then $A G(M)=\emptyset$ or $A G(M)=K_{\alpha}$.

(iv) Let $M$ be a non-simple module with a non-zero socle. Then $A G(M) \neq \emptyset$. In particular, $A G(M) \neq \emptyset$ when $M$ is a non-simple Artinian module.

Proof.

(i) Since $M$ is a semisimple module, we have $M=\oplus_{\alpha \in I} T_{\alpha}$ where, for each $\alpha \in I, T_{\alpha}$ is a simple submodule of $M$. Now let $N$ be an arbitrary non-zero submodule of $M$. Then, by [2, Proposition 9.4], there exist a subset $I^{\prime} \subseteq I$ and a decomposition $N \cong \oplus_{\alpha \in I^{\prime}} T_{\alpha}$. Set $K \cong \oplus_{I \backslash I^{\prime}} T_{\alpha}$. Then $N K \subseteq N \cap K=0$. It follows that $N$ is a vertex.

(ii) Since $M$ is a homogeneous semisimple module, it is clear that $\operatorname{Ann}(M)$ is a maximal ideal of $R$. Hence for every non-zero submodule $N$ of $M$, we have $(N: M)=(0: M)$. We conclude that if $N$ and $K$ are two non-zero distinct submodules of $M$, then $N K=0$, as desired.

(iii) This follows from part (ii) because every prime module with a non-zero socle is homogeneous semisimple (see [9, Corollary 1.9]).

(iv) Suppose that $M$ is not a simple module with $\operatorname{Soc}(M) \neq 0$. Then there exists a minimal submodule $R m$ of $M$, where $m$ is a nonzero element of $M$. Now $(0: m)$ is a maximal ideal of $R$ and we have $(R m)((0: m) M)=0$. This shows that $A G(M) \neq \emptyset$.

Example 3.5. Put $R:=\mathbb{Z}$ and $M:=\oplus_{i \in \mathbb{N}} \mathbb{Z}_{2}$. Since $M$ is a direct sum of isomorphic simple modules, then $M$ is a homogeneous semisimple module. For every non-zero proper submodule $N$ of $M$, we have $(N: M)=\operatorname{Ann}(M)$. Hence every non-zero submodule $N$ and $K$ are adjacent in $A G(M)$.

Proposition 3.6. Let $M$ be a non-simple prime module. Then $A G(M)=K_{\alpha}$, if and only if every non-zero proper submodule of $M$ is adjacent to $M$. 
Proof. The sufficiency is clear.

To see the converse, let $N \in V(A G(M))$. Then there exists a non-zero proper submodule $L$ of $M$ such that $N L=0$. Since $\operatorname{Ann}(M)$ is a prime ideal of $R$, it follows that $(N: M)=\operatorname{Ann}(M)$ or $(L: M)=\operatorname{Ann}(M)$. So every non-zero submodule $M$ is a vertex by Remark 3.1. Now, since $A G(M)$ is a complete graph, every non-zero proper submodule of $M$ is adjacent to $M$.

Theorem 3.7. Consider the following statements.

(i) $\operatorname{Ann}(M)$ is a prime ideal and $M$ is a divisible $R / \operatorname{Ann}(M)$-module.

(ii) Every non-zero proper submodule of $M$ is adjacent to $M$.

(iii) For each ideal $I$ of $R$, we have $I M=M$ or $I M=0$.

(iv) $A G(M)=K_{\alpha}$.

(v) $M$ is a non-simple homogeneous semisimple module.

Then (i) $\rightarrow$ (ii) $\rightarrow$ (iii) $\rightarrow$ (iv) $\rightarrow$ (i). Moreover, if $M$ is a finitely generated module then $(\mathrm{v}) \leftrightarrow(\mathrm{i})$.

Proof. (i) $\rightarrow$ (ii). Let $N$ be a non-zero proper submodule of $M$. We show that $(N: M)=\operatorname{Ann}(M)$. Suppose $r \in(N: M)$ and $r M \neq 0$. Since $M$ is divisible by $R / \operatorname{Ann}(M)$, we have $r M=M$. This implies that $N=M$, a contradiction. Hence, $N$ is adjacent to $M$, as desired.

(ii) $\rightarrow$ (i) and (ii) $\rightarrow$ (iii) are clear.

(iii) $\rightarrow$ (ii). Let $N$ be a non-zero proper submodule of $M$ and $I$ an ideal of $R$. Then $(I M: M)=\operatorname{Ann}(M)$ by hypothesis, where $I M \neq M$. Now we have $(N: M)=((N: M) M: M)=\operatorname{Ann}(M)$. This shows that $N$ is adjacent to $M$, as required.

(ii) $\leftrightarrow$ (iv). Straightforward.

(ii) $\rightarrow$ (v). Let $M$ be a finitely generated $R$-module and let $(N$ : $M)=\operatorname{Ann}(M)$ for every proper submodule $N$ of $M$. Then $M$ is a divisible $R / \operatorname{Ann}(M)$-module. We show that $R / \operatorname{Ann}(M)$ is a field. Suppose not. Then $M$ has a maximal submodule, say $N$. So $(N: M)$ is a maximal ideal $R$. Hence there exists $0 \neq r \in(N: M)$. But $r M=M$ is a contradiction. So $\operatorname{Ann}(M)$ is a maximal ideal and hence $M$ is a homogeneous semisimple module.

(v) $\rightarrow$ (ii). It is clear by Proposition 3.4 (ii). 
Note that an $R$-module $M$ is fully prime (respectively fully semiprime) if each proper submodule of $M$ is prime (respectively semiprime). In [9, Corollary 1.9], it is shown that $M$ is fully prime (respectively, fully semiprime) if and only if is homogeneous semisimple (respectively, co-semisimple module).

Corollary 3.8. Let $R$ be an integral domain with $\operatorname{dim}(R)=1$, and let $M$ be an $R$-module. Then every non-zero proper submodule of $M$ is adjacent to $M$ if and only if one of the following statements hold:

(i) $M$ is a homogeneous semisimple module.

(ii) $M$ is a divisible module.

Proof. Suppose that every non-zero proper submodule of $M$ is adjacent to $M$. Then $\operatorname{Ann}(M)$ is a prime ideal of $R$ and $M$ is a divisible $R / \operatorname{Ann}(M)$-module by Theorem 3.7. If $\operatorname{Ann}(M)=0$, then $M$ is a divisible $R$-module. Otherwise, $\operatorname{since} \operatorname{dim}(R)=1$, it follows that $\operatorname{Ann}(M)$ is a maximal ideal of $R$ so that $(N: M)=\operatorname{Ann}(M)$ for every proper submodule $N$ of $M$. Thus every proper submodule of $M$ is prime by [14, Corollary 1.2]. This means that $M$ is a homogeneous semisimple. Conversely, first we assume that $M$ is a homogeneous semisimple module. Then $\operatorname{Ann}(M)$ is a maximal ideal of $R$ so that every non-zero proper submodule $M$ is adjacent to $M$. In case $M$ is a divisible module, the claim follows from Theorem 3.7.

4. The relationship between $G\left(\tau_{T}^{*}\right)$ and $A G(M)$. A proper submodule $N$ of $M$ is said to be semiprime in $M$ if, for every ideal $I$ of $R$ and every submodule $K$ of $M, I^{2} K \subseteq N$ implies that $I K \subseteq N$. Further, $M$ is called a semiprime module if $(0) \subseteq M$ is a semiprime submodule. Every intersection of prime submodules is a semiprime submodule. A proper ideal $I$ of $R$ is semiprime if, for every ideal $J$ and $K$ of $R, J^{2} K \subseteq I$ implies that $J K \subseteq I$ [17].

Lemma 4.1. Suppose $T$ is a closed subset of $\operatorname{Spec}(M)$ equipped with the natural topology induced from of $\operatorname{Spec}(M)$. Then $T$ and $\operatorname{Spec}(\widehat{M})$ are homeomorphic.

Proof. Let $\phi: \operatorname{Spec}(\widehat{M}) \rightarrow T=V^{*}(\Im(T))$ be defined by $\phi(\widehat{Q})=Q$, where $Q \in \operatorname{Spec}(M)$. Clearly $\phi$ is a bijection map. We show that $\phi$ is a 
continuous map. Let $U=T \cap V^{*}(N)$ be a closed subset of $T$, where $N$ is a proper subset of $M$. Then we have $\phi^{-1}(U)=V^{*}(N \widehat{+\Im(T)})$. We show that $\phi$ is closed. Suppose $U$ is a closed subset of $\operatorname{Spec}(\widehat{M})$. Then $U=V^{*}(\widehat{N})$, where $N \leq M$. It is easy to see that $\phi(U)=V^{*}(N)$.

One may think that since $T$ and $\operatorname{Spec}(\widehat{M})$ are homeomorphic, studying $G\left(\tau_{T}^{*}\right)$ can be reduced to studying $G\left(\tau_{\mathrm{Spec}(L)}^{*}\right)$, where $L$ is a semiprime module. But the following example shows that this is not true.

Example 4.2. Set $R:=\mathbb{Z}, M:=\mathbb{Z} / 12 \mathbb{Z}$, and $T:=\operatorname{Spec}(M)$. Then $G\left(\tau_{T}^{*}\right)=K_{1,2}$ but $G\left(\tau_{\mathrm{Spec}(M / \operatorname{rad}(M))}^{*}\right)=K_{2}$.

Remark 4.3. In fact $G\left(\tau_{T}^{*}\right)$ is a non-empty graph if and only if $\left|E\left(G\left(\tau_{T}^{*}\right)\right)\right| \geq 1$. The following lemma shows that the graph $A G(M)$ also has this property (i.e., $|E(A G(M))| \geq 1$ ) if $M$ is a semiprime module such that it is not a vertex in $A G(M)$.

Lemma 4.4. Assume that $M$ is not a vertex in $A G(M)$. Then $M$ is a semiprime module if and only if for every non-zero submodule $N$ of $M$ and each positive integer $k, N^{k} \neq 0$.

Proof. The necessity is clear.

To see the converse, let $N$ be a submodule of $M$ and let $I$ be an ideal of $R$. Let $I^{2} N=0$ and $I N \neq 0$. Then we have $(I N)^{2}=(I N: M)^{2} M \subseteq$ $I^{2} N=0$, a contradiction. Hence, $M$ is a semiprime module.

Proposition 4.5. The following statements hold.

(i) Suppose $N$ and $L$ are adjacent in $G\left(\tau_{T}^{*}\right)$. Then $\widehat{\sqrt{N}}$ and $\widehat{\sqrt{L}}$ are adjacent in $A G(\widehat{M})$.

(ii) $G\left(\tau_{T}^{*}\right)$ is isomorphic with a subgraph of $A G(\widehat{M})$ or $\left|E\left(G\left(\tau_{T}^{*}\right)\right)\right| \geq 2$.

Proof.

(i) Straightforward.

(ii) Assume that $G\left(\tau_{T}^{*}\right)$ is not isomorphic with a subgraph of $A G(\widehat{M})$. Hence there exist $N, L \in V\left(G\left(\tau_{T}^{*}\right)\right)$ such that $N$ and $L$ are 
adjacent and $N \neq \sqrt{N}$. It follows that $N-L-\sqrt{N}$ is a path of length 2.

Theorem 4.6. The following statements hold.

(i) Let $M$ be a finitely generated module and $G\left(\tau_{T}^{*}\right) \neq \emptyset$. Then $A G(\widehat{M})$ is isomorphic with a subgraph of $G\left(\tau_{T}^{*}\right)$.

(ii) Let $M$ be a fully semiprime module. Then $G\left(\tau_{T}^{*}\right)$ is isomorphic with a subgraph of $A G(\widehat{M})$.

(iii) Let $M$ be a semisimple module and suppose $M$ is not a vertex in $A G(M)$. Then $G\left(\tau_{T}^{*}\right)$ and $A G(\widehat{M})$ are isomorphic.

(iv) Let $M$ be a homogeneous semisimple module. Then $A G(\widehat{M})=$ $K_{\alpha}$, where $\alpha=|\Lambda(\widehat{M})|$ and $G\left(\tau_{T}^{*}\right)=\emptyset$.

Proof.

(i) By [14, Theorem 3.5], every finitely generated top module is multiplication. One can see that if $\widehat{N}$ and $\widehat{L}$ are adjacent in $A G(\widehat{M})$, then $N$ and $L$ are adjacent in $G\left(\tau_{T}^{*}\right)$.

(ii) By [9, Theorem 2.3], $M$ is a co-semisimple module. So

$$
N=\bigcap_{P \in V^{*}(N)} P
$$

where $N<M$. Hence, by Proposition 4.5 (i), it is easy to see that $G\left(\tau_{T}^{*}\right)$ is isomorphic with a subgraph of $A G(\widehat{M})$.

(iii) Let $M$ be a semisimple module and suppose $M$ is not a vertex in $A G(M)$. We show that $M$ is a multiplication module. To see this, let $N$ be a proper submodule of $M$. Then there exists a family $\left\{T_{i}, i \in I\right\}$ of minimal submodules of $M$ such that $N=\oplus_{i \in I} T_{i}$. Now for each $i \in I$, we have $\left(T_{i}: M\right) M=M$ (note that $\left(T_{i}: M\right) M \neq 0$ because $M$ is not a vertex in $A G(M))$. Hence,

$$
N=\bigoplus_{i \in I}\left(T_{i}: M\right) M=\left(\bigoplus_{i \in I}\left(T_{i}: M\right)\right) M .
$$

Thus, $M$ is a multiplication module. It follows that, if $\widehat{N}$ and $\widehat{L}$ are adjacent in $A G(\widehat{M})$, then $N$ and $L$ are adjacent in $G\left(\tau_{T}^{*}\right)$. Since $M$ is a co-semisimple module, by using part (ii), we see that $G\left(\tau_{T}^{*}\right)$ is 
isomorphic with a subgraph of $A G(\widehat{M})$. Hence $G\left(\tau_{T}^{*}\right)$ and $A G(\widehat{M})$ are isomorphic.

(iv) The first assertion follows from Proposition 3.4 (ii). To see the second assertion, $\Im(T)$ is a prime submodule of $M$ (see [9, Corollary 1.9]), thus $G\left(\tau_{T}^{*}\right)=\emptyset$ by Proposition 2.3 (iii).

Example 4.7. Put $R:=\mathbb{Z}$ and $M:=\oplus_{i \in \mathbb{N}} \mathbb{Z} / p_{i} \mathbb{Z}$. Then, by $[\mathbf{6}$, Examples 3.1], $\operatorname{Max}(M)=\operatorname{Spec}(M)=\left\{p_{j} M\right\}=\left\{\oplus_{i \in \mathbb{N}, i \neq j} \mathbb{Z} / p_{i} \mathbb{Z}\right\}$, and $M$ is a top module. $G\left(\tau_{\operatorname{Spec}(M)}^{*}\right)$ is an infinite graph, because every element $\oplus_{i \in \mathbb{N}, i \neq j} \mathbb{Z} / p_{i} \mathbb{Z}$ of $\operatorname{Spec}(M)$ is adjacent to $\mathbb{Z} / p_{j} \mathbb{Z}$. Hence, by Theorem 4.6 (ii), $A G(M)$ is an infinite graph.

Lemma 4.8. Assume that $\emptyset \neq V(A G(\widehat{M}) \subseteq \operatorname{Max}(\widehat{M})$. Then $|T|=2$, $A G(\widehat{M})=K_{2}$, and it is isomorphic with a subgraph of $G\left(\tau_{T}^{*}\right)$.

Proof. Suppose that $\widehat{P}$ is a vertex in $A G(\widehat{M})$ such that $P \in \operatorname{Max}(M)$. Then there exists a non-zero proper submodule $\widehat{Q}$ of $\widehat{M}$ such that it is adjacent to $\widehat{P}$, where, $Q \in \operatorname{Max}(M)$. One can see that $(P: M) \subseteq$ $\left(P^{\prime}: M\right)$ or $(Q: M) \subseteq\left(P^{\prime}: M\right)$ for every $P^{\prime} \in T$. Now since $\widehat{\widehat{M}}$ is a top module, by [14, Theorem 3.5] $P=P^{\prime}$ or $Q=P^{\prime}$. Hence, $V^{*}(P) \cup V^{*}(Q)=T$. It follows that $|T|=2, A G(\widehat{M})$ has only one edge and it is isomorphic with a subgraph of $G\left(\tau_{T}^{*}\right)$.

Proposition 4.9. Assume that $G\left(\tau_{T}^{*}\right) \neq \emptyset$.

(i) If $\widehat{M}$ is a Noetherian $R$-module, then $T=V^{*}\left(P_{1} \cap \cdots \cap P_{n}\right)$, where for each $i(1 \leq i \leq n), P_{i}$ is a vertex.

(ii) If $\widehat{M}$ is an Artinian $R$-module, then $T=V^{*}\left(P_{1} \cap \cdots \cap P_{n}\right)$, where for each $(1 \leq i \leq n), P_{i}$ is a vertex. In particular, $|T|=n$.

Proof.

(i) Since $\widehat{M}$ is a Noetherian module, $\widehat{M}$ has a finite number of minimal prime submodules by [15, Theorem 4.2]. Hence

$$
\operatorname{Spec}(\widehat{M})=V^{*}\left(\widehat{P_{1}}\right) \cup \cdots \cup V^{*}\left(\widehat{P_{n}}\right),
$$


where each $i(1 \leq i \leq n), \widehat{P}_{i}$ is a minimal prime submodule of $\widehat{M}$ and $P_{i}$ is a prime submodule of $M$. So, by Lemma 4.1, we have $T=V^{*}\left(P_{1}\right) \cup \cdots \cup V^{*}\left(P_{n}\right)$. Now the result follows from Lemma 2.8 (i).

(ii) As in the proof of Proposition 2.13 (ii), $\widehat{M} / \operatorname{rad}(\widehat{M})$ is a Noetherian module. So $\widehat{M} / \operatorname{rad}(\hat{M})$ has a finite number of minimal prime submodules. Hence, $\widehat{M}$ has a finite number of minimal prime submodules. So we have $T=V^{*}\left(P_{1}\right) \cup \cdots \cup V^{*}\left(P_{n}\right)$ by part (i). To see the second assertion, we note that, since $\widehat{M} / \operatorname{rad}(\widehat{M})$ is a finitely generated top module, it is a multiplication module by $[\mathbf{1 4}$, Theorem 3.5]. It follows that $\widehat{M} / \operatorname{rad}(\widehat{M})$ is a cyclic Artinian module by [11, Corollary 2.9], and hence, $\operatorname{Spec}(\widehat{M} / \operatorname{rad}(\widehat{M}))=\operatorname{Max}(\widehat{M} / \operatorname{rad}(\widehat{M}))$. So $\operatorname{Spec}(\widehat{M})=\operatorname{Max}(\widehat{M})$. Hence, by the above arguments, we have $|T|=n$, and the proof is completed.

Acknowledgments. We would like to thank the referee for valuable comments and a careful reading of our manuscript.

\section{REFERENCES}

1. D.F. Anderson and P.S. Livingston, The zero-divisor graph of a commutative ring, J. Algebra 217 (1999), 434-447.

2. W. Anderson and K.R. Fuller, Rings and categories of modules, SpringerVerlag, New York, 1974.

3. H. Ansari-Toroghy and F. Farshadifar, Product and dual product of submodules, Far East J. Math. Sci 25 (2007), 447-455.

4. H. Ansari-Toroghy and Sh. Habibi, The Zariski topology-graph of modules over commutative rings, Comm. Algebra. 42 (2014), 3283-3296.

5. H. Ansari-Toroghy and R. Ovlyaee-Sarmazdeh, On the prime spectrum of X-injective modules, Comm. Algebra 38 (2010), 2606-2621.

6. - On the prime spectrum of a module and Zariski topologies, Comm. Algebra 38 (2010), 4461-4475.

7. I. Beck, Coloring of commutative rings, J. Algebra 116 (1988), 208-226.

8. M. Behboodi and M.R. Haddadi, Classical Zariski topology of modules and spectral spaces I, Int. Electr. J. Algebra, 4 (2008), 104-130.

9. M. Behboodi, O.A.S. Karamzadeh and H. Koohy, Modules whose certain ideals are prime, Vietnamese J. Math. 32 (2004), 303-317.

10. M. Behboodi and Z. Rakeei, The annihilating-ideal graph of commutative rings I, J. Alg. Appl. 10 (2011), 727-739.

11. Z.A. El-Bast and P.F. Smith, Multiplication modules, Comm. Alg. 16 (1988), 755-779. 
12. Chin-Pi Lu, The Zariski topology on the prime spectrum of a module, Houston J. Math. 25 (1999), 417-432.

13. R.L. McCasland and M.E. Moore, Prime submodules, Comm. Alg. 20 (1992), 1803-1817.

14. R.L. McCasland, M.E. Moore and P.F. Smith, On the spectrum of a module over a commutative ring, Comm. Alg. 25 (1997), 79-103.

15. R.L. McCasland and P.F. Smith, Prime submodules Noetherian modules, Rocky Mountain J. Math. 23 (1993), 1041-1062.

16. D. Reinard, Graph theory, Grad. Texts Math., Springer, New York, 2005.

17. H.A. Tavallaee and R. Varmazyar, Semi-radicals of submodules in modules, IUST Inter. J. Eng. Sci. 19 (2008), 21-27.

Department of Pure Mathematics, Faculty of Mathematical Sciences, UniVersity of Guilan, P.O. Box 41335-19141 Rasht, Iran

Email address: ansari@guilan.ac.ir

Department of Pure Mathematics, Faculty of Mathematical Sciences, University of Guilan, P.O. Box 41335-19141 Rasht, Iran

Email address: sh.habibi@phd.guilan.ac.ir 